\title{
EGR1 Induces Tenogenic Differentiation of Tendon Stem Cells and Promotes Rabbit Rotator Cuff Repair
}

\author{
Xu Tao ${ }^{a}$ Junpeng Liu ${ }^{a}$ Lei Chen ${ }^{b}$ You Zhou ${ }^{a}$ Kanglai Tang \\ aDepartment of Orthopaedics, Southwest Hospital, Third Military Medical University, Chongqing,

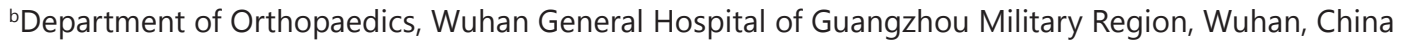

\section{Key Words}

Early growth response 1 (EGR1) • Tendon stem cells (TSCs) • Tenogenic differentiation • Rotator cuff repair

\begin{abstract}
Background/Aims: The rate of healing failure after surgical repair of chronic rotator cuff tears is considerably high. The aim of this study was to investigate the function of the zinc finger transcription factor early growth response 1 (EGR1) in the differentiation of tendon stem cells (TSCs) and in tendon formation, healing, and tendon tear repair using an animal model of rotator cuff repair. Methods: Tenocyte, adipocyte, osteocyte, and chondrocyte differentiation as well as the expression of related genes were determined in EGR1-overexpressing TSCs (EGR1-TSCs) using tissue-specific staining, immunofluorescence staining, quantitative PCR, and western blotting. A rabbit rotator cuff repair model was established, and TSCs and EGR1TSCs in a fibrin glue carrier were applied onto repair sites. The rabbits were sacrificed 8 weeks after repair operation, and tissues were histologically evaluated and tenocyte-related gene expression was determined. Results: EGR1 induced tenogenic differentiation of TSCs and inhibited non-tenocyte differentiation of TSCs. Furthermore, EGR1 promoted tendon repair in a rabbit model of rotator cuff injury. The BMP12/Smad1/5/8 signaling pathway was involved in EGR1-induced tenogenic differentiation and rotator cuff tendon repair. Conclusion: EGR1 plays a key role in tendon formation, healing, and repair through BMP12/Smad1/5/8 pathway. EGR1-TSCs is a promising treatment for rotator cuff tendon repair surgeries.
\end{abstract}




\section{Introduction}

Tendon retear following rotator cuffs repair surgery is a common problem that has been reported in up to $94 \%$ of cases [1]. Various biological approaches have been used to augment surgical repairs to try and improve long-term outcomes of these repair surgeries. These include augmentations with cellular components, extracellular matrix, or various individual growth factors (reviewed in Cheung et al. [1]). However, sufficient improvements in repair in humans have not yet been reported with any of these approaches, and there is a need for more investigations to identify effective biological augmentation strategies.

Among the various options for supplementing rotor cuff repair surgeries, augmentation with growth factors is considered to be the more attractive procedure. Whereas tendon tissue transplants may induce rejection reactions and extracellular matrix transplants may induce inflammation, augmentation with growth factors is not associated with any of these problems. Furthermore, growth factor augmentations are thought to be technically less complicated [1]. Previous studies have shown that growth factors such as bone morphogenic proteins 2, 7, and 12 (BMP-2, BMP-7, and BMP-12, respectively), transforming growth factors $\beta 1$ and $\beta 3$ (TGF- $\beta 1$ and TGF- $\beta 3$, respectively), and fibroblast growth factor (FGF) increase healing rates in animal models. However, even though the size of the tendons tended to increase, the improvements in the repairs with these augmentations were questioned since the strength of the repaired tendons did not increase [1].

The early growth response-1 (EGR1) transcription factor has recently been shown to be involved in tendon differentiation [2]. It was associated with increased collagen formation during tendon cell differentiation in embryo limbs, and it induced expression of the tendon marker scleraxis (SCX) and tendon collagens, including the main tendon collagen Col1a1 [2]. Consistent with the role of EGR1 in tendon differentiation, EGR $1 \%$ mice exhibited weaker tail tendons compared with wild-type mice [3].

In a mouse model of Achilles tendon healing, Guerquin and colleagues showed that 1 week after injury, expression of Egr1 as well as tendon-associated markers started to increase (relative to Egr1 expression in mice with intact Achilles tendon), suggesting that the EGR1 protein is involved in healing [3]. In vitro studies further demonstrated a direct role for EGR1 in mesenchymal stem cells (MSC) differentiation into tendon [3]. These same authors also reported that in a rat model for Achilles tendon injury, implanting EGR1-producing MSCs to the repair site resulted in improved formation of tendon-like tissue at the repair site [3]. Although these results show promise for the use of EGR1-producing MSCs in tendon repair, some researchers have suggested that non-tendon derived stem cells have the potential to differentiate into non-targeted tissues and may, therefore, not be the most favorable cells for use in tendon repair [4].

In the current study we used tendon-derived stem cells (TSCs) to express the EGR1 protein. We hypothesized that EGR1 would drive the differentiation of TSCs into tendon cells and that EGR1-producing TSCs would enhance healing and repair of rotator cuff tears in a rabbit model of this injury.

\section{Materials and Methods}

\section{Ethics Statement}

Twenty-seven New Zealand white rabbits (8-10 week-old males, 3.5-4.0 kg) were used in the study. All animals were treated according to institutional guidelines for laboratory animal treatment and care. All experimental procedures were approved by the Animal Research Ethics Committee of the Third Military Medical University, China (Approval No. SYXK-PLA-20120031).

Isolation and culture of rabbit TSCS

TSCs were isolated from three New Zealand white rabbits and cultured as described previously [5]. Briefly, patellar tendons were dissected and tissue from the middle tendon portion was harvested. The 
peritendinous connective tissue was removed, ground into pieces of approximately $1 \mathrm{~mm} \times 1 \mathrm{~mm} \times 1 \mathrm{~mm}$, and digested with a sterile phosphate-buffered saline (PBS) solution containing $3 \mathrm{mg} / \mathrm{ml}$ type I collagenase (Sigma-Aldrich, St. Louis, MO, USA) and $4 \mathrm{mg} / \mathrm{ml}$ dispase (StemCell technologies Inc., Vancouver, BC, Canada) for $2.5 \mathrm{~h}$ at $37^{\circ} \mathrm{C}$. After centrifuging the suspension at $1,500 \mathrm{x}$ g for $15 \mathrm{~min}$, the supernatant was discarded and the pellet resuspended in growth medium (Dulbecco's modified Eagle's medium [DMEM]; Gibco, Carlsbad, CA, USA) containing 10\% fetal bovine serum, $100 \mathrm{U} / \mathrm{ml}$ penicillin, $100 \mathrm{mg} / \mathrm{ml}$ streptomycin, and $2 \mathrm{mM} \mathrm{L-glutamine} \mathrm{(all} \mathrm{from} \mathrm{Invitrogen,} \mathrm{Carlsbad,} \mathrm{CA,} \mathrm{USA).} \mathrm{Following} \mathrm{dilution} \mathrm{to} \mathrm{different} \mathrm{densities,}$ cells were cultured at $37^{\circ} \mathrm{C}$ in $5 \% \mathrm{CO}_{2}$. Two days following plating, the cells were washed twice with PBS to remove nonadherent cells. On day 7 , they were trypsinized and mixed together as passage 0 cells. Cells from passages 1-3 were used for the following experiments.

\section{Immunofluorescence staining}

TSCs were immuno-stained as described previously [5]. Briefly, TSCs were fixed in $4 \%$ paraformaldehyde/PBS for 30 min. After blocking cells with $2 \%$ mouse serum for $1 \mathrm{~h}$ at room temperature, they were washed with PBS and incubated at room temperature for $3 \mathrm{~h}$ with mouse anti-human primary antibodies (Abcam, Cambridge, MA, USA) against the following stem cell protein markers: octamer-binding transcription factor 4 (Oct-4), stage-specific embryonic antigen-4 (SSEA-4), or nucleostemin. Cells were then washed with PBS and incubated with Cy3-conjugated goat anti-mouse secondary antibody (Abcam) for $30 \mathrm{~min}$ at room temperature. Cells were counter stained with DAPI (Sigma-Aldrich), and examined using fluorescence microscopy.

A similar protocol was adopted to perform immuno-staining of the tenogenic proteins tenomodulin (TNMD) and scleraxis (SCX). Goat anti-human TNMD and SCX primary antibodies (Santa Cruz Biotechnology) were used. Secondary antibodies were FITC-conjugated donkey anti-goat IgG (Abcam) or Cy3-conjugated donkey anti-goat IgG antibodies (Abcam).

\section{Plasmid transfection and RNA interference}

An EGR1-expressing plasmid was transfected into TSCs as described previously [3]. Briefly, the EGR1 coding sequence from the pCMV-EGR1 cDNA clone (OriGene) was cloned into the pCDNA3.1 $1^{+}$vector (Invitrogen) to yield pCDNA-EGR1. TSCs were transfected with pCDNA-EGR1 or the vector alone (control) using the Lipofectamine 2000 transfection reagent (Invitrogen). G418 (800 $\mathrm{\mu g} / \mathrm{ml})$-containing medium was used to select stable transfectants. No morphological differences were observed between the TSCs and TSCs transfected with the empty vector. Additionally, differentiation potential and gene expression were not different between these cells, thus TSCs and TSCs transfected with the empty vector were used interchangeably as controls.

For the RNA interference studies, short-hairpin (shRNA) lentiviral particles targeting the BMP12 and Smad1 genes (Santa Cruz Biotechnology, Santa Cruz, CA, USA) were used to transfect cells according to the manufacturer's instructions.

\section{Induction of TSCs differentiation}

To induce tenogenic differtiation, TSCs and pCDNA-EGR1-transfected TSCs (EGR1-TSCs) were cultured in 6-well plates $\left(2.5 \times 10^{5}\right.$ cells/well) in DMEM (Gibco, Carlsbad, CA, USA) supplemented with $20 \%$ fetal bovine serum, $100 \mathrm{U} / \mathrm{ml}$ penicillin, $100 \mathrm{mg} / \mathrm{ml}$ streptomycin, and $2 \mathrm{mM} \mathrm{L-glutamine} \mathrm{(all} \mathrm{from} \mathrm{Invitrogen,}$ Carlsbad, CA, USA) for up to 14 days with medium change every 2 to 3 days. The expression of differentiationrelated genes was determined by immunofluorescence staining and quantitative PCR.

Adipogenic differentiation was induced by incubating TSCs and EGR1-TSCs in adipogenic induction medium (Lonza, Walkersville, MD, USA) for 7 days and in adipogenic maintenance medium for 2-3 days. After 21 days, cells were washed with PBS, fixed in 10\% neutral buffered formalin, and stained with Oil Red 0 for lipid droplets.

Osteogenic differentiation was performed as described previously [6]. Six-well plates containing $3 \times 10^{3}$ cells $/ \mathrm{cm}^{2}$ were incubated in osteogenic induction medium for 28 days, fixed, and stained with Alizarin Red. For chondrgenic differentiation, a pellet culture system, as described by Riu et al. $[7,8]$ was used. $5 \times 10^{5}$ cells per pellet were incubated in chondrogenic induction medium for 21 days. The pellets were fixed, embedded in paraffin, cut in $5 \mu \mathrm{m}$-thick sections, and stained with Toluidine Blue.

\section{KARGER}


Tao et al.: EGR1-TSCs Promotes Rabbit Rotator Cuff Repair

Table 1. Primers used for quantitative real-time PCR

\begin{tabular}{ll}
\hline Gene & Primers \\
\hline EGR1 & F: 5'- CCCAGCAGCCTTCGCTAACC -3' \\
& R: 5'- CGCTCCTGGCAAACTTTCTTC -3' \\
BMP12 & F: 5'- CCACTTACCACGCCATCTTT -3' \\
& R: 5'- TCTCGTCGTACTGCTTGTAGT -3' \\
SCX & F: 5'- CCTTCTGCCTCAGCAACCAG -3' \\
& R: 5'- GGTCCAAAGTGGGGCTCTCCGTGACT -3' \\
TNMD & F: 5'- AACACTTATACTGGCATCTAT -3' \\
& R: 5'- AACTGTCTCATCTATTTCCTCTTTT -3' \\
TNC & F: 5'- CAGAAGCCTTGGCCATGTG -3' \\
& R: 5'- GCACTCTCTCCCCTGTGTAGGA -3' \\
Collagen I & F: 5'- GACAAGGGTGAGACAGGCGAACAG -3' \\
& R: 5'- CGGGGACAAGAGGGACCAATA -3' \\
PPARG & F: 5'- TCCACAAAAAAAGTAGAAATA -3' \\
& R: 5'- GAACTGGCAGCCCTGAAAGAT -3' \\
RUNX2 & F: 5'- CCGATGGGACCGTGGTT -3' \\
& R: 5'- CAGCAGAGGCATTTCGTAGCT -3' \\
SOX9 & F: 5'- TGAAGAATGGGCAGGCGGAGG -3' \\
& R: 5'- GGGAGTGGTGGGTGGGGTGGT -3' \\
B-actin (internal control) & F: 5'- ATCGTGGGCCGCCCTAGGCA -3' \\
& R: 5'- TGGCCTTAGGGTTCAGAGGGG -3' \\
\hline
\end{tabular}

RNA isolation and quantitative real-time PCR (qRT-PCR)

Gene expression was quantified by qRT-PCR. Total RNA was extracted using TRIzol reagent (Invitrogen) according to the manufacturer's instructions. The first cDNA strand was synthesized using a Superscript III first-strand synthesis kit (Invitrogen) with oligo (dT) primers in a final volume of $20 \mu \mathrm{l}$. qRT-PCR was performed with an ABI 7900 HT system (Applied Biosystems, Foster City, CA, USA) using a QuantiTect SYBR Green RT-PCR kit (Qiagen, Hilden, Germany) [5]. Primers used for qRT-PCR are listed in Table 1.

\section{Protein extraction and Western blotting}

To examine the production of proteins in the BMP12/Smad1/5/8 pathway during tenogenic differentiation, TSCs or EGR1-TSCs were cultured for $0 \mathrm{~h}, 12 \mathrm{~h}, 1 \mathrm{~d}, 3 \mathrm{~d}, 7 \mathrm{~d}$, and $14 \mathrm{~d}$. Cells were washed twice with PBS and lysed in lysis buffer containing a mixture of proteinase inhibitors (Thermo Fisher Scientific Inc., Rockford, IL, USA). To determine levels of tenocyte-related proteins and those in the BMP12/Smad1/5/8 pathway during tendon healing, tendon tissues were digested in a buffer containing $8 \mathrm{M}$ urea, $50 \mathrm{mM}$ Tris$\mathrm{HCl}$ (pH 8), $1 \mathrm{mM}$ dithiothreitol, and $1 \mathrm{mM}$ EDTA. Total protein concentrations were measured using a BCA protein assay kit (Thermo Fisher Scientific Inc.), and equal amounts of extracted proteins ( $30 \mu \mathrm{g} / \mathrm{lane})$ were resolved by SDS-polyacrylamide gel electrophoresis. Proteins were then transferred on to polyvinylidene difluoride membranes, and membranes blocked by incubating with $5 \%$ non-fat milk containing $0.1 \%$ TBSTween for $1 \mathrm{~h}$ at $20^{\circ} \mathrm{C}$. The membranes were then incubated sequentially with primary and secondary antibodies. The following primary antibodies were used: anti-BMP12, anti-phospho-Smad1/5/8, antiSmad, anti-SCX, anti-TNMD, anti-TNC, or anti-Collagen I (all from Santa Cruz Biotechnology). $\beta$-actin was used as an internal control. Proteins were visualized and images captured using a LiCoR Odyssey imager (LI-COR Biosciences, Lincoln, NE, USA).

\section{Chronic rotator cuff tear model}

The methods described by Chung et al. [9] were used to induce rotator cuff tear in 24 mature New Zealand white male rabbits. The supraspinatus tendon was severed in the right shoulder, and sham operations were performed in the left shoulder (control).

\section{Rotator Cuff Tendon Repair}

Six weeks following the induced tear, surgical procedures to repair the injury were performed as described previously [9]. The following procedures were performed in three groups (8 rabbits per group):

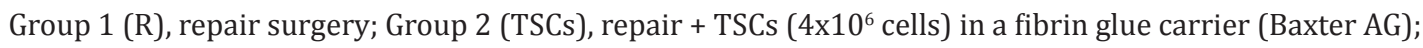
and Group 3 (EGR1-TSCs), repair +EGR1-TSCs $\left(4 \times 10^{6}\right.$ cells) in a fibrin glue carrier. Application of TSCs and EGR1-TSCs in a fibrin glue was as described previously [10]. 


\section{Histological analyses and immunohistochemistry}

Eight weeks after the repair surgical procedure, all rabbits were sacrificed and a specimen of the greater tubercle of the humeral head with the attached supraspinatus tendon of both shoulders of each rabbit was harvested. Three specimens per group were stored at $-80^{\circ} \mathrm{C}$ for mRNA extraction for RT-PCR and for protein extraction for Western blotting. The remaining tissue specimens, which included the supraspinatus tendon and greater tuberosity from the repair site, were fixed in neutral buffered $10 \%$ formalin (pH 7.4), decalcified, and embedded in paraffin blocks. $5 \mu \mathrm{m}$-thick sections were cut in the coronal plane for staining with hematoxylin and eosin (H\&E), Masson's Trichrome and for immunohistochemical analysis of collagen I. For immunohistochemical analysis, the sections were immunolabeled with anti-collagen I primary antibodies at $25^{\circ} \mathrm{C}$ for $1 \mathrm{~h}$. Isotype-matched negative control antibodies (Zymed) were used under the same conditions. The broad-spectrum immunoperoxidase AEC kit (Picture Plus, Zymed) was used to detect immunoactivity.

Semi-quantitative histomorphometric analyses were performed as described previously [9, 11, 12]. The following scoring system was used: 0 (normal), 1 (slightly abnormal), 2 (moderately abnormal), and 3 (maximally abnormal). Five parameters were semiquantitatively used for scoring: vascularity, cellularity, fiber structure, fiber arrangement, and inflammation rate.

Vascularity (area infiltrated by neo-vascular) was scored 0 for $<10 \%$ infiltration; 1 for $10-20 \%$ infiltration; 2 for $20-30 \%$ infiltration; and 3 for $>30 \%$ infiltration. Cellularity (rounding of the nuclei) was scored as follows: 0 for long, spindle-shaped cells; 1 for slight rounding; 2 for moderate rounding; and 3 for severe rounding. Fiber structure was scored as: 0 for continuous, long fiber; 1 for slightly fragmented; 2 for moderately fragmented; and 3 for severely fragmented. Fiber arrangement was scored as 0 for compacted and parallel; 1 for slightly loose and wavy; 2 for moderately loose, wavy and cross to each other; and 3 for no identifiable pattern. Inflammation rate (area infiltrated by inflammation cells) was scored as 0 for $<10 \% ; 1$ for $10-20 \%, 2$ for $20-30 \%$; and 3 for $<30 \%$. Based on this scoring system, a perfectly normal tendon would score 0 and a maximally abnormal tendon would score 15.

\section{Statistical Analysis}

Data were expressed as mean \pm standard deviation (SD). The $t$-test was used to compare between two groups. Multiple comparisons were made using a one-way analysis of variance followed by Fisher's tests. A $\mathrm{p}$ value $<0.05$ was considered to be statistically significant.

\section{Results}

EGR1 induced tenogenic differentiation of TSCS

At passages 3, confluent TSCs isolated from rabbit patellar tendons had the characteristic cobblestone structure of stem cells (Fig. 1A) described previously [5]. Immunofluorescence staining of these TSCs showed they expressed the stem cell marker proteins Oct-4, SSEA-4, and nucleostemin (Fig. 1B, 1C, and 1D, respectively).

Immunofluorescence staining also showed that the EGR1-overexpressing cells (EGR1TSCs) had higher levels of the tenocyte-associated protein markers SCX and TNMD compared with TSCs (Fig. 2A). The effect of EGR1 on the transcription of tenocyte-associated genes was also examined in the EGR1-TSCs. Over-expression of EGR1 resulted in higher levels of the $S C X$, TNMD, TNC, and Col I (encoding Collagen I) transcripts (Fig. 2B), indicating that the effect of EGR1 was at the transcription level of these tenocyte-associated genes.

The specificity of EGR1 in tenocyte differentiation was determined by comparing the histology of TSCs and EGR1-TSCs cultured under adipogenic, osteogenic, and chondrogenic induction conditions. Transcripts for the markers of adipogenesis (PPAR $\gamma$ ), osteogenesis (RUNX2), and chondrogenesis (SOX9) were also quantified. Induction of differentiation of the TSCs in the presence of EGR1 overexpression inhibited adipocyte, osteocyte, and chondrocyte differentiation (Fig. 2C). In addition, PPAR , RUNX2, and SOX9 transcript levels were lower in the EGR1-TSCs compared with TSC cell (Fig. 2D), indicating down-regulation of these genes by EGR1.

\section{KARGER}


Fig. 1. Characterization of rabbit tendon stem cells (TSCs). (A) Cobblestone-like morphology of TSCs in a confluent culture at passages 3 after culturing for 7 days. (B-D) Immunofluorescence staining showing expression of stem cell markers in TSCs. Oct-4 (B, pink dots), SSEA-4 (C, red), and nucleostemin (D, pink dots). Bar: $50 \mu \mathrm{m}$.
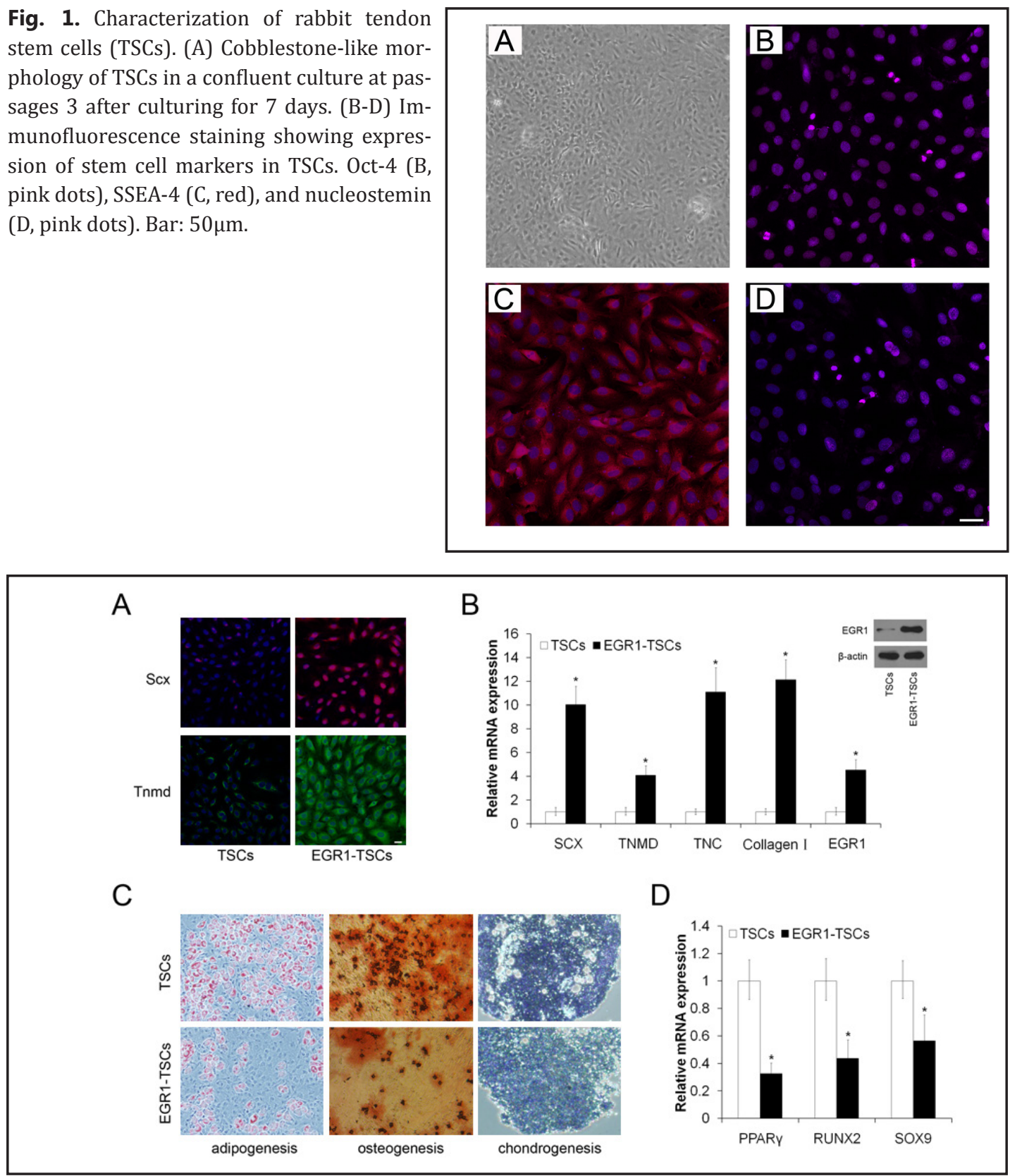

Fig. 2. EGR1 promotes differentiation of rabbit tendon stem cells (TSCs) toward tenocyte lineage in vitro. Rabbit TSCs or EGR1-TSCs were incubated in growth media for 14 days. (A) Immunofluorescence staining of SCX and TNMD protein. Bar: $50 \mu \mathrm{m}$. (B) mRNA levels of the tenocyte-associated genes, SCX, TNMD, TNC, Collagen I quantified by qRT-PCR. Expression efficiency of EGR1 in TSCs and EGR1-TSCs was detected by qRT-PCR and Western blotting (inset). (C) TSCs and EGR1-TSCs were subjected to adipocyte, osteocyte, and chondrocyte differentiation and stained with Oil Red for adipocyte, Alizarin Red for osteocyte, and Toluidine blue for chondrocyte. EGR1 inhibited adipocyte, osteocyte, and chondrocyte differentiation of TSCs. (Magnification of microscopy: 100x). (D) qRT-PCR showed mRNA levels of markers for adipogenesis (PPAR $)$, osteogenesis ( $R U N X 2$ ), and chondrogenesis (SOX9) were downregulated in EGR1-TSCs. Mean \pm standard deviation transcript levels relative to TSCs is shown. ${ }^{*} \mathrm{P}<0.05$ with respect to TSCs.

EGR1 induced tenogenic signaling in TSCs via the BMP12/Smad1/5/8 pathway.

Previous studies had shown that BMP12 activation of the Smad1/5/8 signaling pathway was involved in tenogenic differentiation in cell cultures $[6,13,14]$. To determine if EGR1- 
A

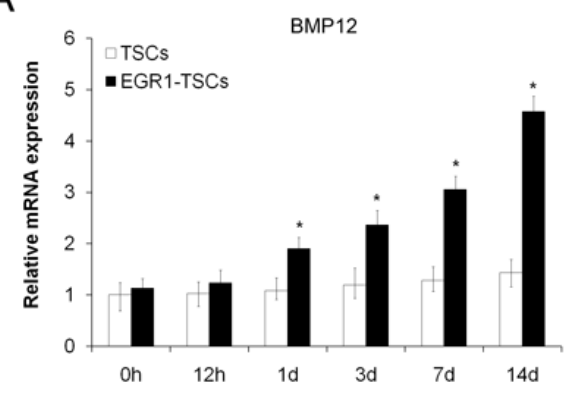

C

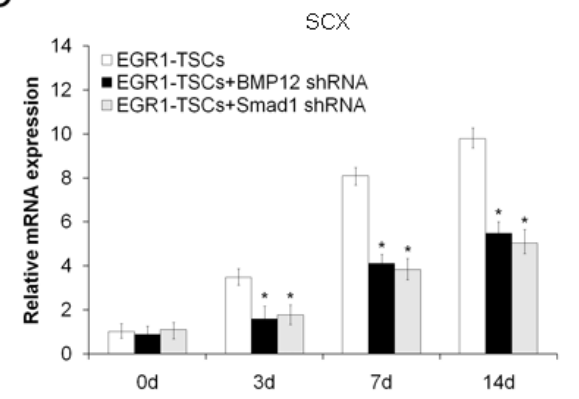

$\mathrm{E}$

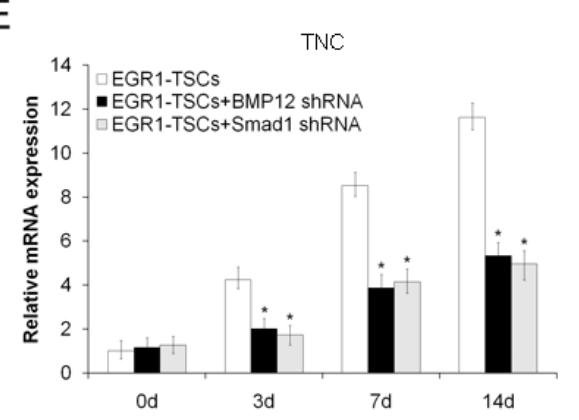

B

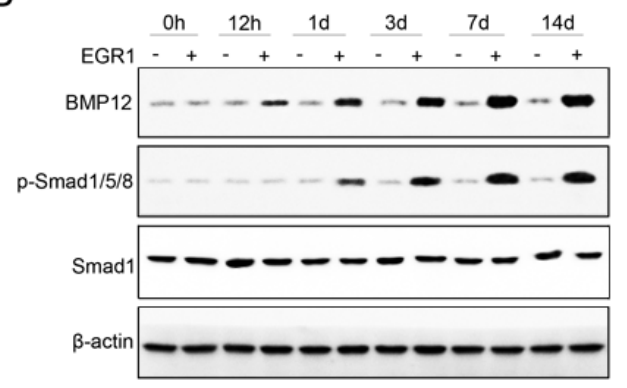

D

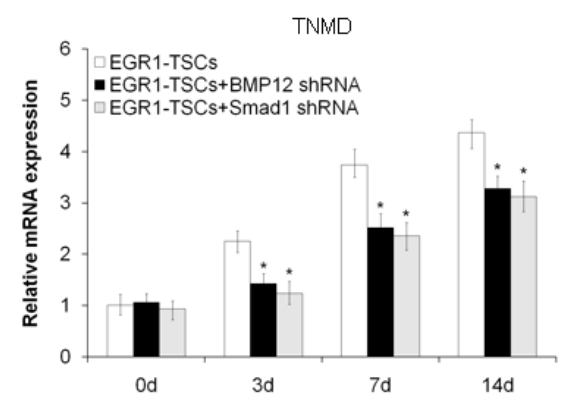

$\mathrm{F}$

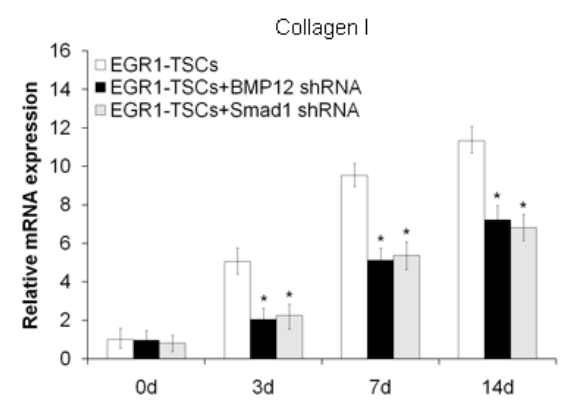

Fig. 3. The BMP12/Smad1/5/8 pathway is involved in EGR1-induced tenogenic differentiation of TSCs. EGR1-overexpressing TSCs were incubated for the indicated number of hours or days. (A) BMP12 mRNA expression was detected by qRT-PCR. ${ }^{*} \mathrm{P}<0.05$ with respect to TSCs. (B) Western blot of BMP12 and total and phosphorylated Smad1 protein levels. (C-D) Tenocyte-associated gene expression in an RNA interference experiment of the BMP12 and Smad1 genes. EGR1-overexpressing TSCs were transfected with BMP12 shRNA or Smad1 shRNA-containing lentivirus vector or were not transfected (control) and incubated for the indicated number of days. Mean \pm standard deviation of transcript levels relative to baseline (T0) levels in EGR-TSCs are shown. (C) SCX mRNA. (D) TNMD mRNA. (E) TNC mRNA. (F) Collagen I mRNA. *P<0.05 with respect to EGR-TSCs for panels 3C-3F.

mediated tenogenic differentiation occurs via the BMP12/Smad1/5/8 signaling pathway in our system, we examined BMP12 and Smad1/5/8 expression. BMP12 mRNA and protein levels increased gradually in a time-dependent manner during tenogenic differentiation in EGR1-overexpressing TSCs but not in TSCs (Fig. 3A and 3B). Similarly, phosphorylated Smad1/5/8 protein levels also increased gradually in a time-dependent manner (Fig. 3B). Total Smad1 protein levels showed no increase during tenogenic differentiation (Fig. 3B). Quantification of the transcripts of the tenocyte-associated genes SCX, TNMD, TNC, and ColI also showed gradual increase in these mRNAs in a time-dependent manner in EGR1-TSCs (Fig. 3C-3F). Expression of BMP12 and Smad1 shRNA in the EGR1-TSCs resulted in lower SCX, 
Fig. 4. EGR1 promotes the formation of tendon-like tissues in a rabbit model for rotator cuff injury. Eight weeks after the repair surgical procedure, rotator cuff tendons from each group were dissected and subjected to histological analysis. Each group had repair surgery in the absence of any implant (R) or in the presence of implants with tendon stem cells in a fibrin glue carrier (TSCs), or EGR1-TSCs in a fibrin glue carrier (EGR1-TSCs). (A) Hematoxylin and eosin (HE) staining and Masson's Trichrome staining (100× magnification). (B) Histology scores of tendon repair at 8 weeks $(n=8$ per group). ${ }^{*} \mathrm{p}<0.05$ with respect to the repair group $(\mathrm{R}) ; \# \mathrm{p}<0.05$ with respect to the TSCs therapy group.

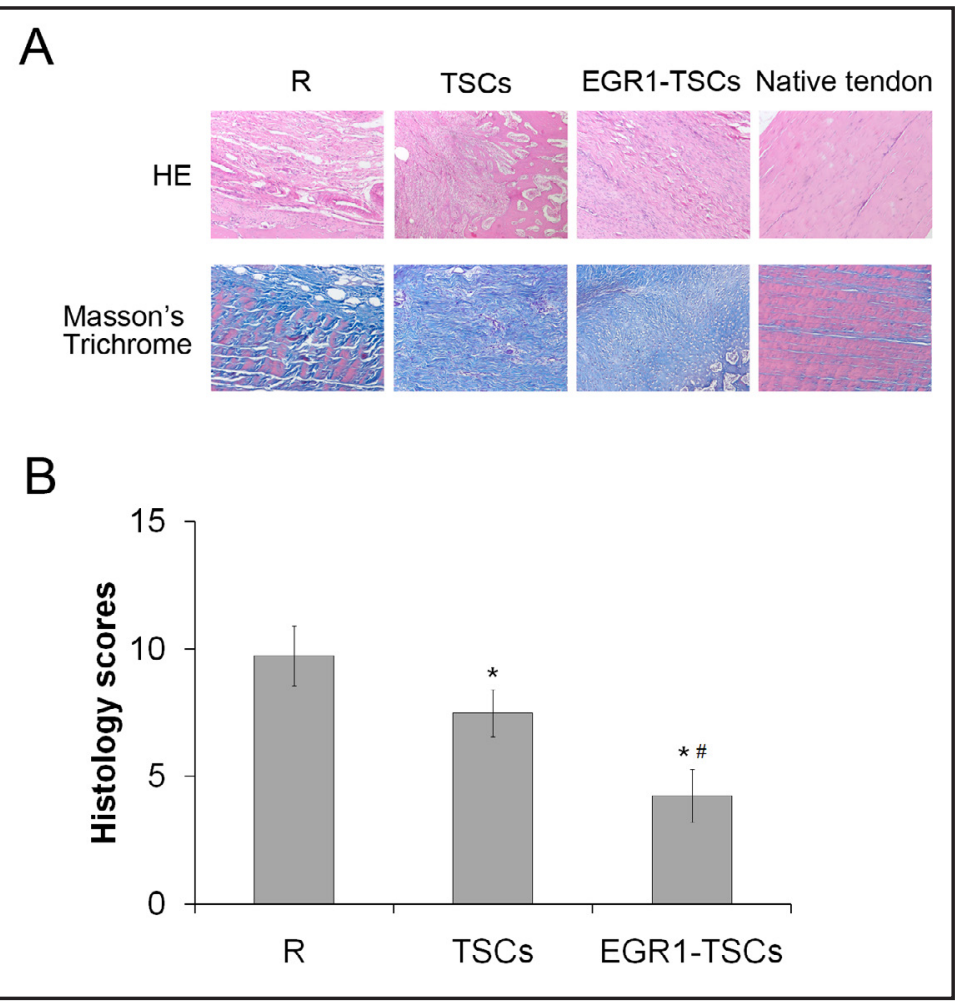

TNMD, TNC, and Collagen I transcript levels (Fig. 3C-3F) compared with the controls lacking these shRNAs, indicating that BMP12 and Smad1 are expressed upstream of the tenocyteassociated genes.

EGR1 promoted tendon repair and activated BMP/Smad1/5/8 signaling in a rabbit model for rotator cuff injury.

No infections or other complications were observed at the surgical site in any of the animals. The peritendinous fibrous reaction was low in all rabbits. There were no adhesions or contractures limiting the range of motion of the shoulder (data not shown). Eight weeks after the detached supraspinatus tendon in rabbits was surgically repaired in the presence or absence of EGR1-TSCs augmentation, tissues were evaluated histologically and molecularly.

At 8 weeks following surgery, the rotator cuff defects in the $\mathrm{R}$ (repair with no augmentation) group were covered with thin fibrous membranes (Fig. 4A), indicating that the defects in our experimental model did not heal spontaneously. The TSCs group showed parallel fibrous tissues, continuously aligned along the load axis at the tendon insertion with small amounts of Sharpey fibers. In the EGR1-TSCs group, collagen fibers were packed in a parallel pattern, with slight splitting between bundles, and many Sharpey fibers were observed at the tendon insertion site (HE, Fig. 4A).

Masson trichrome staining showed collagen-fibril formation in the tendon-bone junction during healing (Fig. 4A). The native tendon had concentrated and orderly collagenfibrils near the tendon-bone junction, whereas the R group had loose collagen-fibrils at the tendon-bone junction and in the tendon. The TSCs group had concentrated collagen-fibrils at the tendon-bone interface near the tendon, and the EGR1-TSCs group had higher collagenfibril expression with some orderly pattern (Fig. 4A).

The mean composite histology scores showed that the repair in the presence of EGRTSCs had better outcome than either repair in the presence of TSCs alone or in the absence of any augmentation (R, Fig. 4B).

Immunohistochemical analysis of repaired tendons showed greatly enhanced staining of Type I collagen protein in the EGR1-TSCs group compared with other groups. (Fig. 5A). 


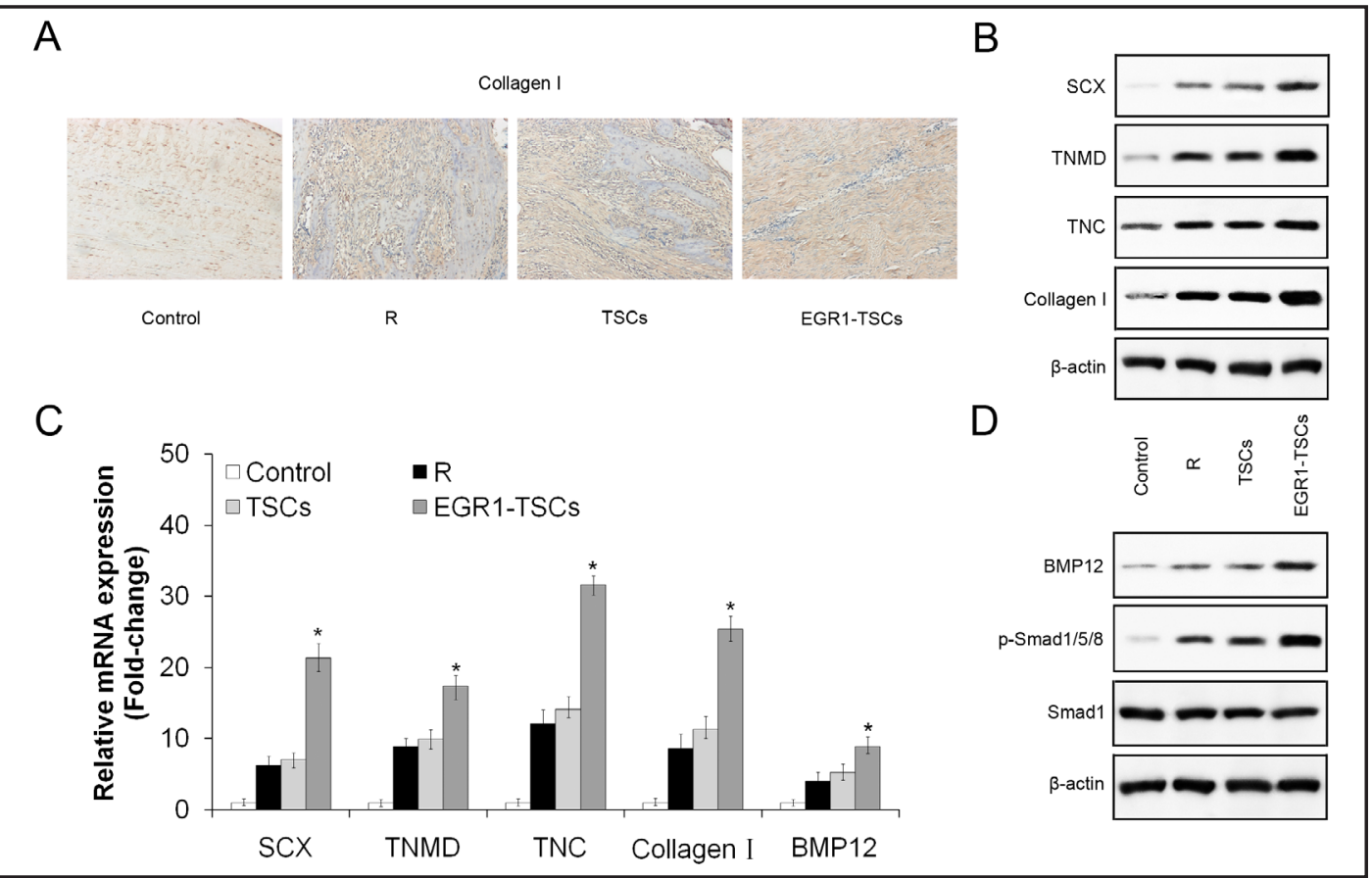

Fig. 5. Induction of tendon cell-related genes and activation of the BMP12/Smad1/5/8 pathway by EGR1 in rabbit tendon stem cells (TSCs) implants in rotator cuff injury. (A) Immunohistochemical staining: Collagen I protein $(100 \times)$. (B) Western blot showing SCX, TNMD, TNC, and Collagen I protein levels. (C) Tendon-associated genes and BMP12 mRNA levels quantified by qRT-PCR; (D) Western blot of total BMP12 and phosphorylated Smad protein levels. Results represent mean \pm standard deviation. ${ }^{*} \mathrm{P}<0.05,{ }^{* *} \mathrm{P}<0.01$ with respect to repair group. " $\mathrm{P}<0.05$ with respect to TSCs.

Although the SCX, TNMD, TNC, collagen, and BMP12 protein levels were higher in the surgically treated animals, the highest concentrations were in rabbits whose surgery was augmented with EGR-TSCs (Fig. 5B). Consistent with this, transcript levels for these genes were highest in the rabbits that had EGR1-TSCs augmented repair surgeries (Fig. 5C). Finally, BMP12 and phosphorylated Smad1 protein levels were higher in the tissues of rabbits treated with EGR1-TSCs than in any of the other groups of rabbits (Fig. 5D), showing that the BMP12-Smad1/5/8 signaling pathway was activated in rabbits during tendon repair, and that this activation was greatest in animals that had EGR1-TSCs implants.

\section{Discussion}

In the current study we showed that in a rabbit model of rotator cuff injury, surgical repair of tendon tear in the presence of EGR1-overexpressing TSCs heals better than repairs performed with implants of TSCs only or with no implants. These results were supported by our in vitro studies, which showed that EGR1 induces specific differentiation of TSCs into tenocytes. EGR1 production in these cells also induced the expression of tendon marker genes SCX, TNMD, TNC, and Collagen1 both in vitro and in vivo.

Our study extends previous works that had demonstrated that EGR1 drives differentiation of MSCs into tendon-lineage [3]. These studies had also shown that implants with EGR1-expressing MSCs improved repair of tendon tears in the rat model of Achilles tendon injury [3].

A study using a rat model of rotator cuff healing had previously shown that animals receiving MSCs producing SCX had better healing compared with those receiving MSCs alone [10]. In that study, tissues repaired in the presence of SCX-producing cells had higher stressKARGER 
to-failure and stiffness values compared with tissues repaired in the absence of the SCXproducing MSCs. Our study showed that SCX levels increased in EGR1 overexpressing cells. Given that EGR1 acts upstream of SCX in the signaling pathway and that it increases SCX as well as other tendon-specific proteins, we would expect similarly improved tendon tissue healing in our rabbit model of rotator cuff repair. However, this will have to be confirmed in additional studies evaluating the biomechanical properties of the regenerated tissue.

Although MSCs have been reported to show promising outcomes for tendon repair, transplantation of these stem cells in tendon repair has also been reported to result in ectopic bone and tumor formation $[15,16,17]$. Thus, stem cells with increased propensity for tendon formation would provide a better option for tendon tear repairs. Liu et al. reported that stem cells derived from tendon tissues may have more advantages (in tendon repair) than stem cells from other sites since the tendon milieu these cells are harvested from may predispose them to tendon generation and to greater potential for expressing tendon-specific markers such as SCX and TNMD [18]. Thus, TSCs may be a better and safer alternative for EGR1 overexpression in tendon repair as they have been reported to have a predisposition to tendon formation [4, 15]. Additionally, TSCs have been reported to have higher clonogenicity and proliferation rates compared with bone marrow stem cells, and this could aid better healing [18].

Finally, consistent with previous studies, we showed that EGR1 induces the BMP12/ Smad1/5/8 pathway during tendon differentiation in vitro and during tendon tear healing in vivo. The current study, nevertheless, has some limitations. First, it was limited by the difficulty of replicating human disease in an animal model, and our results should be interpreted with caution regarding clinical application, because of the differences in the anatomy of the shoulder and the intrinsic healing potential between rabbits and humans. Second, we did not track the TSCs and EGR1 expression in vivo and thus cannot be entirely certain that the TSCs stayed in the desired site and that these cells overexpressed EGR1. However, expression of EGR1 by transduced TSCs was confirmed with RT-PCR and Western blotting in vitro. Since the TSCs and EGR1-TSCs-treated groups showed a significant effect on tendon-bone healing in this study, we believe that TSCs were present at the repair site and that these cells overexpressed EGR1. Future studies to track the EGR1-TSCs after injection using labeled cells still need to clarify their differentiation status and potential contribution to tendon healing. Finally, we studied the status of tendon-to-bone healing only at a midterm period until 8 weeks after repair and had no information on further changes at a later postoperative period. We selected 8 weeks based on previous studies $[9,19]$, but there certainly could be more mechanical and biological changes after 8 weeks. Therefore further study with a longer postoperative observation period may be needed.

In conclusion, our studies demonstrated that EGR1-TSCs could be useful for augmenting rotator cuff tendon repair surgeries. Additional studies are required to evaluate the longterm quality of these tendon repairs.

\section{Acknowledgements}

This study was supported by the National Nature Science Foundation of China (grant nos. 81230040).

\section{Disclosure Statement}

None.

\section{References}

$\rightarrow 1$ Cheung EV, Silverio L, Sperling JW: Strategies in biologic augmentation of rotator cuff repair: A review. Clin Orthop Relat Res 2010;468:1476-1484. 
Tao et al.: EGR1-TSCs Promotes Rabbit Rotator Cuff Repair

2 Lejard V, Blais F, Guerquin MJ, Bonnet A, Bonnin MA, Havis E, Malbouyres M, Bidaud CB, Maro G, Gilardi-Hebenstreit P, Rossert J, Ruggiero F, Duprez D: Egr1 and egr2 involvement in vertebrate tendon differentiation. J Biol Chem 2011;286:5855-5867.

- 3 Guerquin MJ, Charvet B, Nourissat G, Havis E, Ronsin O, Bonnin MA, Ruggiu M, Olivera-Martinez I, Robert N, Lu Y, Kadler KE, Baumberger T, Doursounian L, Berenbaum F, Duprez D: Transcription factor egr1 directs tendon differentiation and promotes tendon repair. J Clin Invest 2013;123:3564-3576.

4 Zhang J, Li B, Wang JH: The role of engineered tendon matrix in the stemness of tendon stem cells in vitro and the promotion of tendon-like tissue formation in vivo. Biomaterials 2011;32:6972-6981.

-5 Zhang J, Wang JH: Characterization of differential properties of rabbit tendon stem cells and tenocytes. BMC Musculoskelet Disord 2010;11:10.

6 Shen H, Gelberman RH, Silva MJ, Sakiyama-Elbert SE, Thomopoulos S: Bmp12 induces tenogenic differentiation of adipose-derived stromal cells. PLoS One 2013;8:e77613.

7 Rui YF, Lui PP, Li G, Fu SC, Lee YW, Chan KM: Isolation and characterization of multipotent rat tendonderived stem cells. Tissue Eng Part A 2010;16:1549-1558.

8 Rui YF, Lui PP, Wong YM, Tan Q Chan KM: Bmp-2 stimulated non-tenogenic differentiation and promoted proteoglycan deposition of tendon-derived stem cells (tdscs) in vitro. J Orthop Res 2013;31:746-753.

-9 Chung SW, Song BW, Kim YH, Park KU, Oh JH: Effect of platelet-rich plasma and porcine dermal collagen graft augmentation for rotator cuff healing in a rabbit model. Am J Sports Med 2013;41:2909-2918.

10 Gulotta LV, Kovacevic D, Packer JD, Deng XH, Rodeo SA: Bone marrow-derived mesenchymal stem cells transduced with scleraxis improve rotator cuff healing in a rat model. Am J Sports Med 2011;39:12821289.

11 Chen J, Yu Q Wu B, Lin Z, Pavlos NJ, Xu J, Ouyang H, Wang A, Zheng MH: Autologous tenocyte therapy for experimental achilles tendinopathy in a rabbit model. Tissue Eng Part A 2011;17:2037-2048.

$\checkmark 12$ Chen JM, Willers C, Xu J, Wang A, Zheng MH: Autologous tenocyte therapy using porcine-derived bioscaffolds for massive rotator cuff defect in rabbits. Tissue Eng 2007;13:1479-1491.

13 Mazerbourg S, Sangkuhl K, Luo CW, Sudo S, Klein C, Hsueh AJ: Identification of receptors and signaling pathways for orphan bone morphogenetic protein/growth differentiation factor ligands based on genomic analyses. J Biol Chem 2005;280:32122-32132.

14 Wang QW, Chen ZL, Piao YJ: Mesenchymal stem cells differentiate into tenocytes by bone morphogenetic protein (bmp) 12 gene transfer. J Biosci Bioeng 2005;100:418-422.

15 Lui PP, Rui YF, Ni M, Chan KM: Tenogenic differentiation of stem cells for tendon repair-what is the current evidence? J Tissue Eng Regen Med 2011;5:e144-163.

16 Harris MT, Butler DL, Boivin GP, Florer JB, Schantz EJ, Wenstrup RJ: Mesenchymal stem cells used for rabbit tendon repair can form ectopic bone and express alkaline phosphatase activity in constructs. J Orthop Res 2004;22:998-1003.

17 Tasso R, Augello A, Carida M, Postiglione F, Tibiletti MG, Bernasconi B, Astigiano S, Fais F, Truini M, Cancedda R, Pennesi G: Development of sarcomas in mice implanted with mesenchymal stem cells seeded onto bioscaffolds. Carcinogenesis 2009;30:150-157.

18 Lui PP, Wong OT: Tendon stem cells: Experimental and clinical perspectives in tendon and tendon-bone junction repair. Muscles Ligaments Tendons J 2012;2:163-168.

19 Hirose K, Kondo S, Choi HR, Mishima S, Iwata H, Ishiguro N: Spontaneous healing process of a supraspinatus tendon tear in rabbits. Arch Orthop Trauma Surg 2004;124:374-377. 